\title{
Confirmation De Ségrégation, Au Moyen Du BC1, De L'un Des Deux Loci Codant Pour La Couleur Du Germe Chez Le Cocotier Nain à Abidjan (Côte d'Ivoire)
}

\author{
Sidibé Daouda, \\ Unité de Formation et de Recherche de Biosciences, \\ Université Félix Houphouët Boigny, Côte d'Ivoire \\ Issali Auguste Emmanuel, \\ Ministère de la Recherche Scientifique et de l'Innovation Technologique, \\ Université Marien Ngouabi,Congo-Brazzaville \\ Luc Baudouin, \\ CIRAD, Unité Mixte de Recherche 1334 AGAP, \\ Avenue Agropolis, 34398 Montpellier Cedex5, France \\ Roland Bourdeix, \\ Bios - Unité Mixte de Recherche, Amélioration Génétique et Adaptation des \\ Plantes tropicales et méditerranéennes - Bâtiment, France \\ Sokouri Didier Paulin, \\ Unité de Formation et de Recherches de Biosciences, \\ Université Félix Houphouët Boigny, Côte d'Ivoire \\ Serge Tra Bi Doubi, \\ Centre National de Recherche Agronomique, 01BP 1740, Abidjan \\ N'guetta Assanvo Simon-Pierre, \\ Unité de Formation et de Recherches de Biosciences, \\ Université Félix Houphouët Boigny, Côte d'Ivoire
}

Doi:10.19044/esj.2020.v16n24p88 ～URL:http://dx.doi.org/10.19044/esj.2020.v16n24p88

\section{Résumé}

Pour confirmer la ségrégation de l'un des deux loci codant pour la couleur du germe chez les cocotiers nains, le Back cross 1 : NJM x (NJM x $\mathrm{GOA}^{+}$) a été réalisé dans le cadre d'un projet de cartographie du génome du cocotier. Un donneur NJM x GOA ${ }^{+}$(Nain jaune de Malaisie x Grand Ouest Africain $^{+}$) et une receveuse NJM (Nain jaune de Malaisie) ont été impliqués dans le BC1. Les statistiques descriptives, le test U de Mann-Withney et le test du Khi-deux de conformité ont été appliqués. Pour un total de 1034 noix semences germées, les résultats ont montré que sur 5 ségrégations testées, seule la 1:1 vérifie la conformité des données observées à celles théoriques au 
seuil de risque de $5 \%$. Ceci suggère que le couple d'allèles Vert/Jaune codant pour l'expression phénotypique y afférente ségrége selon les lois de la génétique classique. Ces résultats s'apparentent à ceux de Bourdeix. Fort malheureusement, son analyse génétique était sous-tendue par de nombreuses hypothèses. De plus, les semences de noix étaient issues de pollinisations libres de pieds d'hybrides F1. Dans les deux cas, ce sont les germes issus des noix F2 germées qui ont été analysées. La ségrégation $1: 1$ issu du $\mathrm{BC} 1$ : NJM x (NJM x $\mathrm{GOA}^{+}$) suggère un contrôle monofactoriel du marqueur vert/jaune. Ce marqueur pourrait être introgressé dans les génotypes d'intérêt avant d'être utilisé, par la suite, pour la sélection précoce en germoir.

Mots clés : Back cross, Confirmation, Locus, Marqueur vert/jaune, Ségrégation 


\title{
Confirmation of Segregation, By Means of BC 1, of One of the Two Loci Encoding for the Color of the Germ in Dwarf Coconut Palm at Abidjan (Côte d'Ivoire)
}

\section{Sidibé Daouda,}

Unité de Formation et de Recherche de Biosciences, Université Félix Houphouët Boigny, Côte d'Ivoire

Issali Auguste Emmanuel,

Ministère de la Recherche Scientifique et de l'Innovation Technologique,

Université Marien Ngouabi,Congo-Brazzaville

\section{Luc Baudouin,}

CIRAD, Unité Mixte de Recherche 1334 AGAP, Avenue Agropolis, 34398 Montpellier Cedex5, France

\section{Roland Bourdeix,}

Bios - Unité Mixte de Recherche, Amélioration Génétique et Adaptation des Plantes tropicales et méditerranéennes - Bâtiment, France

Sokouri Didier Paulin,

Unité de Formation et de Recherches de Biosciences, Université Félix Houphouët Boigny, Côte d'Ivoire

Serge Tra Bi Doubi,

Centre National de Recherche Agronomique, 01BP 1740, Abidjan

N'guetta Assanvo Simon-Pierre,

Unité de Formation et de Recherches de Biosciences, Université Félix Houphouët Boigny, Côte d'Ivoire

\begin{abstract}
To confirm the segregation of one of the two loci coding for the color of the germ in dwarf coconut palms, back cross 1: MYD x (MYD x WAT $\left.{ }^{+}\right)$ was carried out as part of a coconut genome mapping project. MYD $x \mathrm{WAT}^{+}$ (Malayan Yellow Dwarf x West African Tall ${ }^{+}$) donor and MYD (Malayan Yellow Dwarf) receiving parents were involved in BC1. Descriptive statistics, the Mann-Withney's U test and the Chi-square conformity test were applied. For a total of 1034 seed nuts germinated, results showed that out of 5 segregations tested, only the 1: 1 verifies the conformity of the observed data to the theoretical ones at the threshold of 5\%. This suggests that the alleles
\end{abstract}


pair green / yellow encoding the related phenotypic expression segregates according to the laws of classical genetics. These results are similar to those of Bourdeix. Unfortunately, the genetic analysis of the latter was underpinned by many assumptions. In addition, the seed nuts were obtained from free pollinations of F1 hybrid. In both cases, only germs from the germinated F2 nuts were analysed. The segregation 1:1 from BC1: MYD x (MYD x WAT $\left.{ }^{+}\right)$ suggests a monofactorial control of the green / yellow marker. This marker could be introgressed into the useful genotypes before being used, thereafter, for early selection in the seed bed.

Keywords: Back cross, Confirmation, Green / yellow marker, Locus, Segregation

\section{Introduction}

Le cocotier est une plante monocotylédone pérenne de la famille des Arecaceae (Guyot, 1992). Le déterminisme génétique de la transmission du caractère "couleur du germe" chez le cocotier nain a déjà été étudié par Bourdeix (1988). Il a montré que le caractère "couleur du germe" est sous le contrôle de deux loci ségrégeant indépendamment. En d'autres termes, il a proposé la ségrégation indépendante des deux loci Vert / Jaune et Rouge / Jaune codant pour l'expression de ce caractère.

Cependant, cette analyse a été sous-tendue par de nombreuses hypothèses notamment les diverses incertitudes induites par la composition du nuage pollinique et les taux d'autogamie variables selon les hybrides (Bourdeix, 1988). De même, le plan de croisement utilisé ne pouvait permettre une analyse génétique rigoureuse. En somme, de notre point de vue, l'essai PBGC4 utilisé par Bourdeix, ne se prêtait pas de bout en bout à une telle analyse.

Pour cette analyse, nous postulons qu'une des cinq ségrégations proposées fait correspondre les résultats théoriques à ceux observés. A ce jour, aucune étude n'a été conduite pour vérifier ou confirmer l'approche de Bourdeix sur la ségrégation indépendante des deux loci Vert / Jaune et Rouge / Jaune codant pour l'expression de ce caractère. La présente étude visait à confirmer ou infirmer, au moyen du BC1 : NJM x (NJM x GOA ${ }^{+}$), la ségrégation de l'un des deux loci, codant pour le marqueur Vert / Jaune, proposée par Bourdeix (1988).

\section{Matériel et méthodes}

\subsection{Site d'expérimentation}

La présente étude a été réalisée à la station de recherche Marc Delorme du Recherche Agronomique (CNRA) de Port-Bouët à Abidjan (Côte d'Ivoire). Cette station est située à $12 \mathrm{Km}$ de la route de Grand-Bassam à 
gauche du corridor de Gonzagueville (Figure 1). Elle est localisée à $5^{\circ} 14^{\prime}$ et $5^{\circ} 15^{\prime}$ de latitude Nord et $3^{\circ} 54^{\prime}$ et $3^{\circ} 55^{\prime}$ de longitude Ouest.

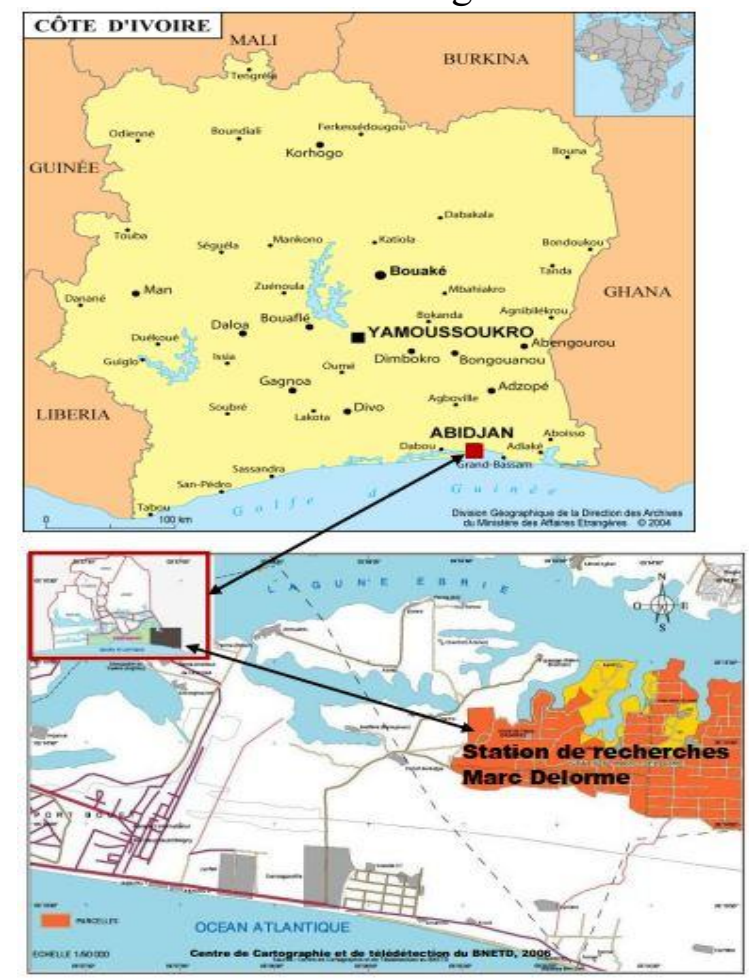

Figure 1: Situation du site d'expérimentation

Les noix semences ont été produites du 2 avril 2013 au 04 avril 2014. Le germoir a été conduit du 10 mars au 07 septembre 2014.

Le climat de la station est du type équatorial. Il est caractérisé par quatre saisons dont deux saisons pluvieuses et deux saisons sèches d'étendues inégales. La grande saison sèche s'étend de décembre à mars alors que la grande saison pluvieuse couvre les mois d'avril à juillet. La petite saison sèche dure d'août à septembre tandis que la petite saison pluvieuse s'étend d'octobre à novembre. La pluviométrie est caractérisée par une précipitation moyenne annuelle de 1673,99 $\mathrm{mm}$ et les températures moyennes mensuelles varient de $24,62{ }^{\circ} \mathrm{C}$ à $27,63{ }^{\circ} \mathrm{C}$ (Source : Service météo de la station de recherches Marc Delorme).

Les sols sont de types tertiaires et constitués de sables grossiers présentant des teneurs en matière organique faible. Ce sont des sols riches en éléments nutritifs dont 690 ppm de phosphore (TIE BI, 1984).

\subsection{Matériel végétal et technique}

Le matériel végétal utilisé pour l'analyse du déterminisme génétique du marqueur vert/jaune de la couleur du germe a été constitué des individus 
BC1 : NJM x (NJM x GOA $\left.{ }^{+}\right)$. Le NJM est un cultivar nain beaucoup utilisé dans les activités de recherche et souvent rencontré en milieu paysan (Assa et al., 2007). Le PB $121^{+}$ou NJM x GOA ${ }^{+}$demeure 1'hybride le plus productif et les plus vulgarisé au monde (Bourdeix et al., 2005). Les remarquables performances de cet hybride (précocité et potentiel de production élevé) ont été décrites à plusieurs reprises (Frémond, 1971 ; De Nucé de Lamothe,1975 ; Sangaré, 1980).

Les données, basées sur l'observation des couleurs, ont été collectées sur les individus au germoir. Les couleurs ont été observées dans la semaine suivant l'apparition du germe. C'est la période où les colorations sont les plus discriminantes (Bourdeix, 1988).

Pour la préparation de la planche du germoir, une houe, une ficelle utilisée pour la délimitation du germoir, une pelle et un râteau ont été utilisés. Un cahier de recherche et un stylo à bille ont été aussi utilisés pour enregistrer toutes les activités.

\subsection{Méthodes}

Les données soumises à l'analyse génétique du marqueur vert/jaune de la couleur du germe ont été obtenues en deux phases. Dans la première phase, les noix semences ont été produites. Dans la seconde étape, les noix semences récoltées ont été mises en germoir.

\subsubsection{Préparation des arbres, fécondations et récolte des noix}

Sur 240 cocotiers nains jaunes malais (NJM) de la parcelle 132, 198 ont été choisis. Ces derniers, utilisés comme arbres-mères, ont été d'abord déchargés. Puis, lorsqu'ils ont émis les inflorescences, celles-ci ont été émasculées puis fécondées par les pollens provenant de 4 cocotiers PB $121^{+}$. Ceux-ci ont été choisis sur 195 cocotiers PB $121^{+}$de la parcelle 115 . Chaque arbre-mère a été fécondé par le pollen provenant de chaque arbre-père. Les fécondations ont été faites progressivement. Lorsque les noix sont arrivées à maturité, notamment entre 9 et 10 mois, elles ont été récoltées.

\subsubsection{Mise en germoir et collecte des données}

Les noix récoltées ont été groupées, transportées et stockées sur l'aire de la pépinière. Deux semaines plus tard, les germoirs préparés à cet effet ont été ensemencés avec les noix semences selon la méthode préconisée par l'IRHO (Wuidart, 1981). Il y a eu au total 11 planches de germoir. Les planches de germoir avaient les dimensions suivantes : $3 \mathrm{~m}$ de largeur et $10 \mathrm{~m}$ de longueur. Ces planches étaient séparées entre elles par des allées de 0,5 m, en forme de drain. Chaque planche contenait uniquement des noix d'une même date de récolte. 
Une semaine suivant l'apparition du germe, les données ont été collectées à base de l'observation de la couleur du germe. Cette observation a été du type binaire, basée sur la présence de 2 phénotypes, notamment le phénotype [jaune] et celui [vert]. Les germes de phénotype [jaune] ont été notés "1" alors que ceux exprimant le phénotype [vert] ont été notés "0" (Figure 2).

\subsubsection{Mise en place de l'expérimentation}

Plusieurs planches de germoirs ont été mises en place progressivement, en fonction des groupes de récolte. Dans chaque germoir, les noix ont été semées horizontalement les uns à côté des autres et en quinconce. Au total, pour cet essai, 1787 noix ont été ensemencées (Figure 3).

\subsection{Analyse statistique des données}

Les logiciels SPSS version 22.0 et Xlstat version 2016 ont été utilisés pour le traitement statistique des données collectées. Les statistiques descriptives et la comparaison des médianes selon le test U de Mann-Withney au seuil de significativité de $5 \%$ ont permis la description de la population $\mathrm{BC} 1$ générée. Toutefois, la méthode non-paramétrique du khi-deux de conformité de Pearson au seuil de significativité de $5 \%$, elle, a permis la réalisation de l'analyse génétique de la couleur du germe.

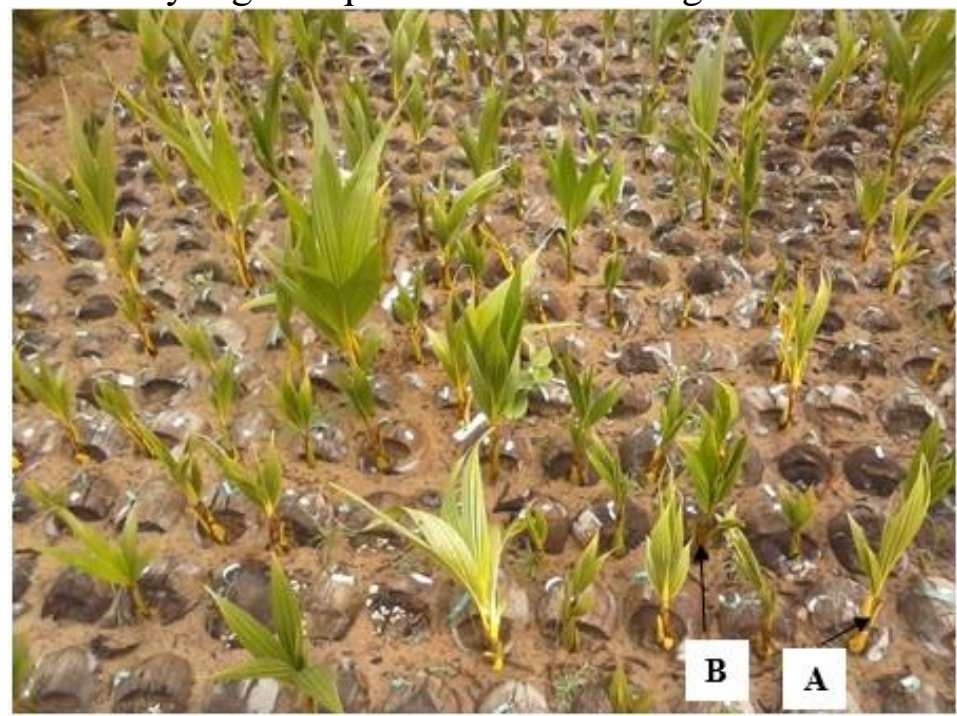

Figure 2: Stade d'observation des 2 phénotypes de la couleur du germe. A, le phénotype [jaune]. B, le phénotype [vert]. 


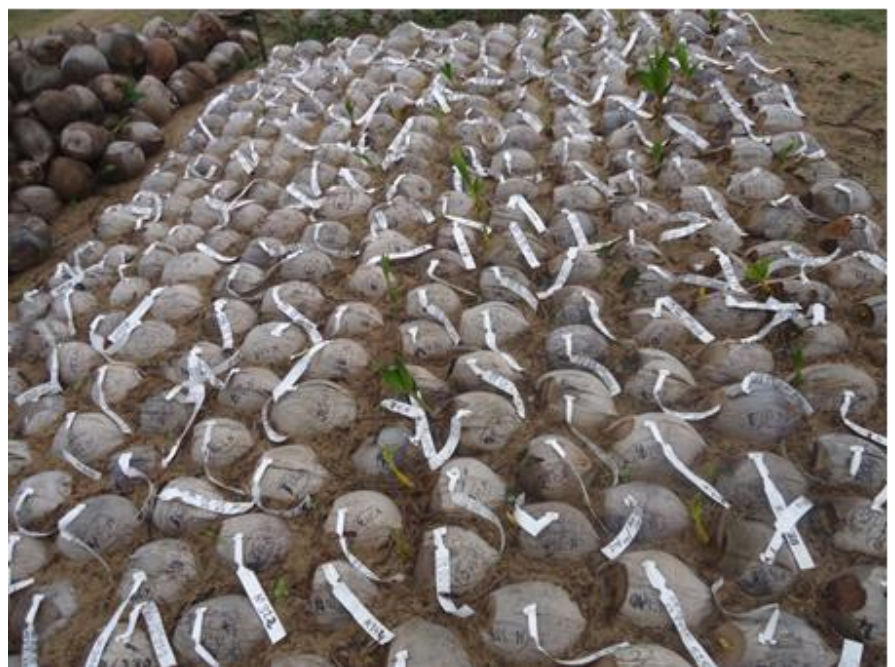

Figure 3: Ensemencement de germoirs avec les noix semences $\mathrm{BC} 1: \mathrm{NJM} \times\left(\mathrm{NJM}_{\mathrm{x} \mathrm{GOA}}{ }^{+}\right)$.

\section{Résultats}

2.1. Description de la population en ségrégation $\mathrm{BC1}: \mathrm{NJM} \times \mathrm{NJM} \times$

GOA selon les phénotypes vert et jaune codé par le locus $\mathrm{J} / \mathrm{j}$ à partir des statistiques élémentaires, de boîtes à moustache et de la comparaison des médianes en utilisant le test $U$ de Mann-Withney

Pour les statistiques élémentaires, les moyennes des phénotypes vert, codé 0 , et jaunes, codé 1 , paraissent numériquement différents (Moyenne vert $/ 0=63,900$ jours ; Moyenne jaune $/ 1=65,480$ jours $)$. Il en va de même pour les 2 médianes y afférentes (Médiane vert/0 $=56,500$ jours ; Médiane jaune $/ 1=58,000$ jours). Les écarts entre chaque modalité du délai de sortie des germes et la moyenne de chaque phénotype sont élevés $(\mathrm{CV}$ vert $/ 0=48$, $39 \% ; \mathrm{CV}$ vert $/ 0=47,30 \%>20 \%$ (Falconer, 1974)). Néanmoins, l'asymétrie et l'aplatissement sont compris dans l'intervalle allant de [-2;+2] laissant penser à une normalité de distributions des variables des 2 échantillons (Tableau I).

Par ailleurs, la représentation dudit délai de sortie des germes au moyen des boîtes à moustache a montré la différence numérique des moyennes et médianes des 2 échantillons. Les charnières basse et haute de la boîte à moustache du phénotype vert (0) sont en déçà de celle du phénotype jaune (1). Les moustaches basses sont en déçà de celles hautes. Le délai de 164 jours pour la sortie de germes de phénotype vert (0) correspondant aux observations 113 et 116. Ce délai représente une valeur extrême (Figure 4).

Concernant la comparaison des médianes, 492 germes de phénotype vert (0) ont été comptés, contre 542 de phénotypes jaune (1). Des rangs moyens et somme de rangs respectivement 509,15 et 250501 pour le phénotype vert (0) ainsi que 525,08 et 284594 pour le phénotype jaune (1) ont 
été calculés (Tableaux II et III). Ils ne sont pas significativement différents (pvaleur $=0,391)$.

Tableau I: Paramètres de tendance centrale et de dispersion pour la description de la

\begin{tabular}{|c|c|c|c|c|c|}
\hline Variable & Code & Phénotype & Paramètres & Statistiomes & CV (\%) \\
\hline \multirow[t]{10}{*}{ Délai } & 0 & vert & Moyenne & 63,990 & 48,39 \\
\hline & & & Médiane & 56,500 & \\
\hline & & & Ecart type & 30,964 & \\
\hline & & & Asymétrie & 0,819 & \\
\hline & & & Kurtosis & $-0,061$ & \\
\hline & 1 & jaune & Moyenne & 65,480 & 47,30 \\
\hline & & & Médiane & 58,000 & \\
\hline & & & Ecart type & 30,969 & \\
\hline & & & Asymétrie & 0,784 & \\
\hline & & & Kurtosis & $-0,183$ & \\
\hline
\end{tabular}

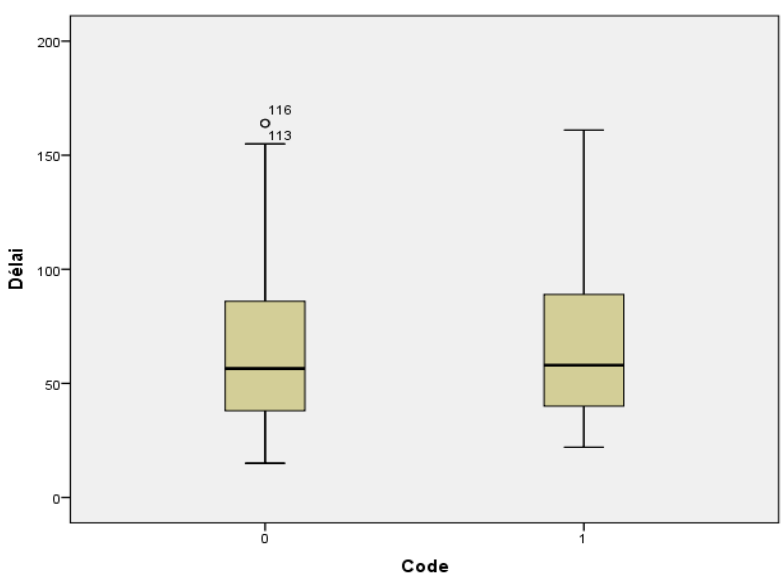

Figure 4 : Représentation du délai de sortie de germes des descendances de croisement $B C 1$ : NJM $\times\left(N J M \times \mathrm{GOA}^{+}\right)$exprimant le phénotype vert (0) et jaune (1) au moyen de boîtes à moustache.

Tableau II: Effectif des phénotypes des germes verts et jaunes et rangs moyens afférents.

\begin{tabular}{ccccc}
\hline Variable & Code & $\mathbf{N}$ & $\begin{array}{c}\text { Rang } \\
\text { moyen }\end{array}$ & $\begin{array}{c}\text { Somme des } \\
\text { rangs }\end{array}$ \\
\hline Délai & 0 & 492 & 509,15 & 250501 \\
& 1 & 542 & 525,08 & 284594 \\
& Total & 1034 & & \\
\hline
\end{tabular}


Tableau III: Significativité de la différence entre les médianes du délai de sorties des germes des noix semences de phénotype vert (0) et jaune (1) selon le test U de MannWithney à 2 échantillons indépendants.

\begin{tabular}{cc}
\hline Tests et statistique afférente & Délai \\
\hline U de Mann-Whitney & 129223 \\
Z & $-0,857$ \\
p-valeur & 0,391 \\
\hline
\end{tabular}

\subsection{Tests de ségrégations}

Parmi les 5 ségrégations testées fournissant la conformité entre les données observées et celles théoriques de 2 classes phénotypiques, seule la ségrégation 1:1 au seuil de probabilité de $5 \%$ l’a vérifiée (Tableau IV).

Tableau IV: Recherche de la ségrégation la plus probable grâce au test paramétrique du Khi-deux de conformité.

\begin{tabular}{|c|c|c|c|c|c|c|c|c|}
\hline $\begin{array}{l}\text { Ségrégations } \\
\text { phénotypiques }\end{array}$ & $\begin{array}{l}\text { Variables } \\
\text { nominales }\end{array}$ & Phénotypes & $\begin{array}{c}\text { Effectifs } \\
\text { observés* }\end{array}$ & $\begin{array}{c}\text { Effectifs } \\
\text { théoriques* }\end{array}$ & Résidus & Sources & $\begin{array}{c}\text { Valeurs } \\
\text { associées }\end{array}$ & Significativité \\
\hline \multirow{3}{*}{ 1:1 } & 0 & [vert] & 492 & 517 & -25 & $\chi^{2} \mathrm{cal}^{*}$ & 2,418 & \\
\hline & 1 & [jaune] & 542 & 517 & 25 & ddl & 1 & \\
\hline & Total & - & 1034 & 1034 & & p-value & 0,120 & NS \\
\hline \multirow{3}{*}{$3: 1$} & $\mathbf{0}$ & [vert] & 492 & 258,5 & 233,5 & $\chi_{\mathrm{cal}}^{2}$ & 281,224 & \\
\hline & $\mathbf{1}$ & [jaune] & 542 & 775,5 & $-233,5$ & ddl & 1 & \\
\hline & Total & - & 1034 & 1034 & & p-value & 0,000 & $\mathbf{S}$ \\
\hline \multirow{3}{*}{ 9:7 } & 0 & [vert] & 492 & 452,4 & 39,6 & $\chi_{\text {cal }}^{*}$ & 6,170 & \\
\hline & 1 & [jaune] & 542 & 581,6 & $-39,6$ & ddl & 1 & \\
\hline & Total & - & 1034 & 1034 & & p-value & 0,013 & $\mathbf{S}$ \\
\hline \multirow{3}{*}{$13: 3$} & 0 & [vert] & 492 & 193,9 & 298,1 & $\chi^{2} \mathrm{cal}^{*}$ & 564,224 & \\
\hline & 1 & [jaune] & 542 & 840,1 & $-298,1$ & ddl & 1 & \\
\hline & Total & - & 1034 & 1034 & & p-value & 0,000 & $\mathbf{S}$ \\
\hline \multirow{3}{*}{$15: 1$} & O & [vert] & 492 & 64,6 & 427,4 & $\chi^{2} \mathrm{cal}^{*}$ & 3014,716 & \\
\hline & 1 & [jaune] & 542 & 969,4 & $-427,4$ & ddl & 1 & \\
\hline & Total & - & 1034 & 1034 & & p-value & 0,000 & $\mathbf{S}$ \\
\hline
\end{tabular}

Légende : $\chi^{2}$ cal ${ }^{*}:$ Khi-deux calculé; $\chi^{2}$ th $:$ valeur du Khi-deux théorique 3,841. Effectifs observés: Effectifs observés pour la couleur du germe. Effectifs théoriques: Effectifs théoriques pour la couleur du germe. ddl : nombre de degrés de liberté correspondant à 2 classes phénotypiques $(n-1=1)$. Significativité* $: N S:$ non significatif ; $S:$ significatif.

\section{Analyse génétique relative à la ségrégation $1: 1$}

Les plants au germoir ont été directement assimilés à une F2 pour l'analyse génétique.

\section{-Observations}

Sur 1787 noix ensemencées, 1034 ont germé. Les 1034 noix germées ont été hétérogènes relativement à leur couleur de germe. Une partie des plants 
a exprimé une couleur jaune, correspondant au phénotype [jaune] alors que l'autre a montré une couleur verte, illustré par le phénotype [vert].

\section{- Calcul des proportions phénotypiques}

Effectif total $=103$

$$
\begin{aligned}
& \text { [jaune }]=\frac{542 \times 100}{1034}=52,42 \%, \text { soit environ } 1 / 2 \\
& {[\text { vert }]=\frac{492 \times 100}{1034}=47,58 \%, \text { soit environ } 1 / 2}
\end{aligned}
$$

L’observation de la couleur du germe a révélé que sur les 1034 germes analysés, la moitié des germes a exprimé le phénotype [jaune] et l'autre moitié le phénotype [vert]. Ce qui nous donne la ségrégation $1 / 2-1 / 2$ au niveau des phénotypes.

\section{-Interprétation génétique}

La ségrégation $1 / 2-1 / 2$ ou $1: 1$ nous permet de conclure que :

- Le marqueur Vert / Jaune est gouverné par un couple d'allèles avec dominance complète de l'un des allèles.

- Les géniteurs croisés sont l'un hétérozygote et l'autre homozygote récessif.

- Les Nains étant assimilés à des lignées pures, le géniteur receveur est homozygote récessif. Le géniteur donneur est donc hétérozygote.

\section{-Choix des symboles}

Le géniteur mâle PB $121^{+}$, de couleur verte, provient du croisement entre le NJM (jaune) et le $\mathrm{GOA}^{+}$(vert). Ce qui signifie que le [vert] est dominant sur le [jaune]. Le couple d'allèles en jeu est $\mathrm{J} / \mathrm{j}$.

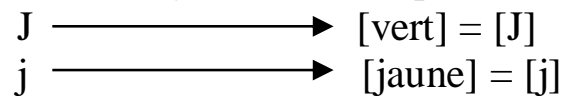

La conformité des effectifs expérimentaux à ceux théoriques attendus au seuil de significativité de $5 \%$ est vérifiée, selon le test de conformité du Khi-deux. Il en ressort que le Khi-deux calculé $\left(\chi^{2}\right.$ cal $\left.=2,418\right)$ est inférieur au Khi-deux lu $\left(\chi^{2}\right.$ lu $\left.=3,841\right)$ au seuil de probabilité de $5 \%$. En conséquence, les écarts constatés entre les effectifs expérimentaux et ceux théoriques sont attribuables au hasard. La significativité du test de conformité du Khi-deux ouvre la voie à la vérification des ségrégations obtenues en $\mathrm{BC} 1$. 
-Vérification des ségrégations obtenues en BC1

Création de PB 121 ${ }^{+}$: $\quad$ 요 $\mathrm{P} 1 \mathrm{x} \overbrace{}^{\Uparrow} \mathrm{P} 2$

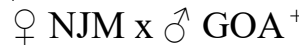

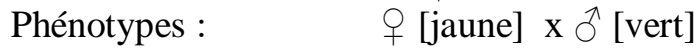

Génotypes :

$$
\stackrel{\mathrm{j}}{\overline{\mathrm{j}}} \times \delta \frac{\mathrm{J}}{\mathrm{J}}
$$

Gamètes :

Hybride PB $121^{+}$:

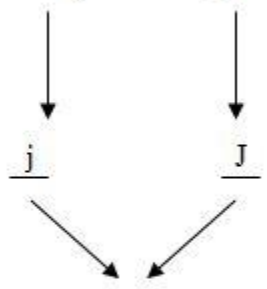

[vert]

$$
\frac{\mathrm{J}}{\overline{\mathrm{j}}}
$$

Création de $\mathrm{BC1}$ :

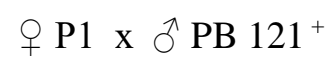

+ $\mathrm{NJM} \times \overbrace{}^{\Uparrow} \mathrm{PB} 121^{+}$

Phénotypes :

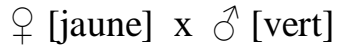

\begin{tabular}{|c|c|c|}
\hline Gamète $q$ Gamète $\delta$ & $1 / 2 \quad \mathrm{~J}$ & j \\
\hline $1 \mathrm{j}$ & $1 / 2 \stackrel{\mathrm{J}}{\overline{\mathrm{j}}}[\mathrm{Jj}]=[$ vert $]$ & $1 / 2 \frac{\mathrm{j}}{\overline{\mathrm{j}}} \quad[\mathrm{jij}]=[\mathrm{jaune}]$ \\
\hline
\end{tabular}

Génotypes : $\quad$ se référer à $1: \stackrel{\mathrm{j}}{\overline{\mathrm{j}}} \times \frac{\mathrm{J}}{\overline{\mathrm{j}}}$ nent (Tableau V)

Tableau V: Vérification des résultats de BCl à travers l'échiquier de croisement

Bilan : 1/2 des germes exprime le phénotype [vert]

$1 / 2$ des germes exprime le phénotype [jaune]

Les résultats expérimentaux sont conformes aux résultats théoriques dans le cas d'un couple d'allèles avec dominance complète de l'un des allèles.

\section{Discussion}

\subsection{Ségrégation de la population}

Un échantillon de 1034 individus régénérés à partir de 1787 noix semences produites pour créer une population de cartographie $\mathrm{BC} 1$ : NJM $\mathrm{x}$

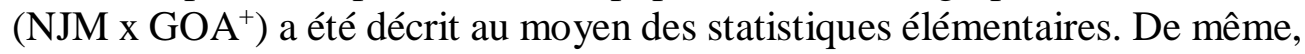
cinq ségrégations fournissant deux classes phénotypiques ont été testées. Bourdeix (1988) a dépouillé les données de germoir issues de la germination de noix semences F2 de pollinisation libres de 3 croisements. Il a obtenu la 
ségrégation $1: 1$. Les présents résultats ont fourni la même ségrégation que celle obtenue par Bourdeix suggérant que le marqueur de la couleur du germe Vert/jaune est sous le contrôle d'un couple d'allèles $\mathrm{J} / \mathrm{j}$.

Les médianes des phénotypes vert (0) et jaune (1) de la couleur du germe sont statistiquement identiques. Donc, les valeurs partageant les délais de sortie de germes de phénotype vert et jaune en deux parties égales sont statistiquement identiques ; même si des différences numériques existent. Le test U de Mann-Withney a été préféré en raison de la non-normalité des distributions des deux échantillons soumis à l'analyse. Ceci est attesté par les coefficients de variation des deux échantillons qui sont supérieures à $20 \%$, quand bien même l'asymétrie et l'aplatissement soient compris dans l'intervalle $[-2 ;+2]$. De même, la non-normalité des distributions a été vérifiée au moyen des tests Kolmogorov-Smirnov et Shapiro-Wilks. Ce test a été aussi utilisé par Djoman (2016). Ceci en prélude de l'égalité de proportions ou fréquences devant régir les tests de ségrégation.

Les délais médians de sortie de germes de la population $\mathrm{BC} 1$ : $\mathrm{NJM} \mathrm{x}$

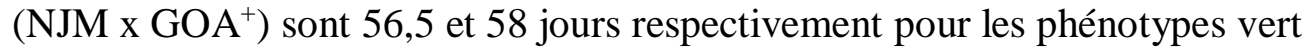
et jaune, contre 112,5 jours et 59,5 jours respectivement pour le GOA et le NJM (Ngbra, 2017). Ces 2 derniers parents expriment des phénotypes vert/brun et jaune. Les 492 et 542 descendants BC1 : NJM x (NJM x GOA $\left.{ }^{+}\right)$ ayant révélé les phénotypes vert et jaune ont montré des valeurs médianes de sortie de germes comprises dans la gamme des valeurs parentales $\mathrm{GOA}^{+}$et NJM. Néanmoins, le délai de sortie des germes phénotype jaune des descendances $\mathrm{BC} 1$, notamment 58 jours, est identique à celui du parent $\mathrm{NJM}$, 59,5 jours. L'homozygotie des descendants $\mathrm{BC} 1$ exprimant le phénotype jaune pour la couleur du germe pourrait expliquer une telle identité. La récessivité de l'allèle $\mathrm{j}$ en serait la cause principale. A contrario, les descendants $\mathrm{BC} 1$ ayant exprimé le phénotype vert pour la couleur de leurs germes, ont enregistré un délai de sortie de germes très en déçà de celui du parent $\mathrm{GOA}^{+}$. Ici, il semblerait que l'hétérozygotie des descendants $\mathrm{BC} 1$ montrant le phénotype vert pour la couleur du germe associé à la présence dans leur génome des allèles de précocité de sortie de germes du NJM seraient à l'origine de la réduction dudit délai. Il serait toujours bon d'impliquer le NJM dans les programmes d'hybridation requérant la création des descendances à sortie précoce de germes.

\subsection{Ségrégations et classes phénotypiques}

Parmi les cinq ségrégations testées, fournissant deux classes phénotypiques pour chaque éventualité, seule la 1:1 a montré une conformité entre les données théoriques et celles observées au seuil de risque de $5 \%$. Bourdeix (1988) a obtenu des résultats similaires. Néanmoins, les données de Bourdeix ont été obtenues à partir des noix semences récoltées sur des pieds 
F1 de trois croisements NJM x NVE, NRM x NJM et NVE X NRM pollinisés librement auxquels a été adjoint le témoin génétique NJM. Ces noix semences F2 ont été enfouies dans les planches de germoir. Les germes émergés de ces noix semences ont été comptés et classés en 2 phénotypes vert et jaune. Par ailleurs, une corrélation positive existe entre la couleur de la noix au stade juvénile et celle du germe au germoir (Bourdeix et al., 2005). Cette recherche de ségrégation a aussi été utilisée par Sinan (2016). Ce dernier a obtenu une ségrégation 13: 3 montrant une conformité entre les données théoriques et celles observées au seuil de risque de $5 \%$.

Le marqueur Vert / Jaune est sous la dépendance d'un couple d'allèles avec dominance complète de l'un des allèles. Le phénotype [jaune] observé chez le NJM est récessif. En effet, l'expression du phénotype [vert] au niveau des germes des individus $\mathrm{PB} 121^{+}$ou $\mathrm{NJM} \times \mathrm{GOA}^{+}$et les ségrégations phénotypiques obtenues en $\mathrm{BC} 1$ (1:1) l'ont confirmé. La confirmation a surtout été apportée par le test Khi-deux qui a établi la conformité des données expérimentales à celle théoriques au niveau de confiance de $95 \%$.

\subsection{La conformité entre les observations}

La conformité entre les observations et le déterminisme proposé pourrait être considérée comme très satisfaisante. En effet, excepté le taux élevé de noix non germées, notamment, $57 \%$, aucun cas d'illégitimité n'a été observé. Cela témoigne aussi des bonnes conditions d'application des techniques de fécondations artificielles utilisées. Ces résultats s'opposent à ceux de Baudouin et Pomiès (2013) qui ont obtenu $3 \%$ d'illégitimité. Ce taux est acceptable puis que le seuil admis en Côte d'Ivoire est de 5\% (Bourdeix, 1988).

Les résultats de cette étude confirment donc, en partie, ceux de Bourdeix (1988). En effet, Bourdeix a travaillé sur des croisements impliquant 3 différentes couleurs (jaune, vert, rouge) correspondant aux marqueurs Vert / Jaune et Rouge / Jaune.

La connaissance de la ségrégation $1: 1 \mathrm{du}$ couple d'allèles $\mathrm{J} / \mathrm{j}$ codant pour le phénotype jaune permettrait l'introgression du phénotype jaune dans les génotypes d'intérêt pour les sélectionner précocement au germoir.

$\mathrm{Du}$ point de vue pratique, le caractère "couleur du germe" peut permettre un contrôle a postériori de la légitimité de certaines fécondations artificielles ou pollinisations dirigées (Bourdeix, 1988). Ce caractère peut être aussi utilisé comme marqueur génétique et/ou biochimique de sélection précoce lors d'une production de masse d'hybrides (Bourdeix et al., 2005).

\section{Conclusion}

L'analyse génétique visant la confirmation ou l'infirmation, au moyen du $\mathrm{BC} 1$, la ségrégation de l'un des 2 loci, en l'occurrence le marqueur Vert / 
Jaune, proposée par Bourdeix a été réalisée. Pour ce faire, les noix semences ont été d'abord produites selon le plan de croisement BC 1. Elles ont été ensuite récoltées puis mises en germoir. Une semaine après l'apparition du germe, les données basées sur l'observation de 2 phénotypes, notamment le phénotype [jaune] et celui [vert], ont été collectées puis dépouillées.

Les ségrégations phénotypiques obtenues à partir du $\mathrm{BC} 1$ ont montré un contrôle monofactoriel. Et, le couple d'allèles contrôlant le marqueur Vert / Jaune est $\mathrm{J} / \mathrm{j}$. La connaissance de la ségrégation $1: 1$ du couple d'allèles $\mathrm{J} / \mathrm{j}$ codant pour le phénotype jaune permettrait l'introgression du phénotype jaune dans les génotypes d'intérêt pour les sélectionner précocement au germoir. Par ailleurs, le déterminisme génétique sur l'autre marqueur (Rouge / Jaune) et la relation génétique entre les deux marqueurs devraient être réalisés pour confirmer ou infirmer le déterminisme proposé par Bourdeix.

\section{Remerciements}

Les auteurs tiennent à remercier les responsables du Centre de coopération Internationale en Recherche Agronomique pour le Développement (CIRAD) pour l'initiation du projet de cartographie du génome du cocotier, de Bioversity International (BI) pour le financement dudit projet et du Centre National de Recherche Agronomique (CNRA) pour avoir abrité les expérimentations.

\section{References :}

1. Assa R. R., Konan J. L., Agbo N., Prades A. et Nemlin J. (2007). Caractéristiques physico-chimiques de l'eau des fruits de quatre cultivars de cocotier (cocos nucifera 1.) en côte d'ivoire. Agronomie Africaine 19 (1) : p 41-51.

2. Baudouin L. et Pomiès V. (2013). Analyses microsatellites effectuées en 2012 sur du matériel prélevé au CNRA, Station Marc Delorme. Rapport d'analyse des échantillons collectés à la Station Marc DELORME du CNRA, 11p.

3. Bourdeix R. (1988). Etude du déterminisme génétique de la couleur du germe chez le cocotier Nain. Oléagineux, 3 (10) : p 371-374.

4. Bourdeix R., Konan J. L. et N'cho Y. P. (2005). Cocotier. Guide des variétés traditionnelles et améliorées. Edition diversiflora, Montpellier (France), 104p.

5. De Nucé de Lamothe et Rognon F. (1975). L'hybride Port-Bouet 121. Nouveaux résultats (bilingue fr.-angl.). Oléagineux, 30, $\mathrm{N}^{\circ} \mathrm{IJ}$, p. 457 465.

6. Djoman N. S. (2016). Effet en autofécondation de la pose du sac, de son enlèvement et de la nouaison sur l'expression de la fructification chez 24 hybrides F2 Nain x Nain de cocotier : Cocos nucifera L. 
(Arecaceae), Mémoire de Master 2, UFR Biosciences,Université Félix Houphouët Boigny, Abidjan, Côte d'Ivoire, 58 p.

7. Falconer D.S. (1974). Introduction à la génétique quantitative. Masson et $\mathrm{C}^{\mathrm{ie}}, 120$, Boulevard Saint-Germain Paris VI ${ }^{\mathrm{e}}$, pp 227-232.

8. Frémond Y. et De Nucé de Lamothe M. (1971). Caractéristiques et production du cocotier hybride « Nam Jaune Malaisie X Grand Ouest Africain 1), Oléagineux, 26, $\mathrm{N}^{\circ}$ 7, p. 459-464

9. Guyot M. (1992). Systématique des angiospermes : référence à la flore du Togo. Edition financée par la Mission Française de Coopération et d'Action Culturelle de Lomé. Diffusion auprès de la bibliothèque de l’Université du Benin, Togo, p 172-173.

10. N'gbra K. F. (2017). Ressemblance au germoir et à la pépinière des hybrides $\mathrm{PB}_{113^{+}}$et $\mathrm{PB} 121^{+}$de cocotier : Cocos nucifera $\mathrm{L}$. (Arecaceae). Mémoire de Master 2, UFR Biosciences, Université Félix Houphouët Boigny, Abidjan, Côte d'Ivoire, 56 p.

11. Rognon F. (1972). Production du matériel végétal cocotier : Sélection des hybrides en germoir. Oléagineux, 27e année, no 4. P. 203-204.

12. Sangaré A. et Rognon F. (1980). Production de l'hybride Port-Bouet 121 (bilingue fr.-angl.). Oléagineux, 35, $\mathrm{N}^{\circ} 2$, p. 79-82.

13. Sinan K. I. (2016). Déterminisme génétique de la couleur de la bourre et typologie des plantules hybrides F2 Nain x Nain de cocotier : Cocos nucifera L. (Arecaceae). Mémoire de Master 2, UFR Biosciences, Université Félix Houphouët Boigny, Abidjan, Côte d'Ivoire, 48 p.

14. Tié Bi Y. (1984). Contribution à l'étude des sols sableux de la basse Côte d'Ivoire, cultivée en cocotiers et définition des seuils d'utilisation de la fumure phosphatée. Thèse de Doctorat 3ème cycle. Université de Cocody (Abidjan, Côte d'Ivoire), 182p.

15. Wuidart W. (1981). Production de matériel végétal cocotier. Tenue d'un germoir. Oléagineux, Vol. 36, nº 6: 305-309. 